\title{
Screening for gestational diabetes mellitus by two step method and the pregnancy outcome: a study in Indian women
}

\author{
Suguna Srinivasan, Vijayalakshmi Palanisamy*
} Department of Obstetrics and Gynaecology, Shri Sathya Sai Medical College and Research Institute, Ammapettai
Kancheepuram, Tamilnadu, India

Received: 20 July 2019

Accepted: 26 July 2019

\section{*Correspondence:}

Dr. Vijayalakshmi Palanisamy,

E-mail: vijayalakshmi01434@gmail.com

Copyright: (C) the author(s), publisher and licensee Medip Academy. This is an open-access article distributed under the terms of the Creative Commons Attribution Non-Commercial License, which permits unrestricted non-commercial use, distribution, and reproduction in any medium, provided the original work is properly cited.

\section{ABSTRACT}

Background: In recent times, gestational diabetes is becoming more common Worldwide and complications are seen in fetal development, growth, labour and delivery due to maternal hyperglycemia. Gestational Diabetes Mellitus(GDM) is associated with adverse maternal and fetal outcomes. Among South Asian Population, Indian Women are at high risk of developing carbohydrate intolerance during pregnancy. Hence this study is undertaken to screen for gestational diabetes mellitus by two step method in Indian Women.

Methods: This study was done in 153 nondiabetic pregnant women of gestational age 24-28 weeks, excluding diabetes mellitus diagnosed prior to pregnancy. Two step method was followed in the present study and GDM was diagnosed and the pregnancy outcomes were noted.

Results: In our study the prevalence of GDM is $8.1 \%$ by two step method. Most of the women diagnosed with GDM were of age 26-30 yrs (41.66\%). Among the women who were diagnosed as GDM, (58.33\%) cases required Insulin along with diet control. In our study $33.33 \%$ had vaginal delivery, $8.33 \%$ had Emergency LSCS and 58.33\% had Elective LSCS.

Conclusion: Indian Women have high prevalence of GDM, hence universal screening is essential to diagnose GDM, which will improve the pregnancy outcome.

Keywords: Gestational diabetes mellitus, Glucose challenge test, Lower segment cesarean section, Oral glucose tolerance test

\section{INTRODUCTION}

Gestational diabetes mellitus (GDM) is defined as Carbohydrate intolerance of variable Severity with onset or recognition during Pregnancy, irrespective of treatment with diet or Insulin. Gestational diabetes is associated with adverse maternal and fetal outcomes. ${ }^{1}$ With rapid urbanization, changing diets, decreasing physical activity, the trend towards delayed marriage and older maternal age as well as the growing epidemics of obesity and type 2 diabetes, the prevalence of GDM may very well be on the rise. ${ }^{2}$ Among the ethnic groups in South Asia, Indian Women have the highest incidence of
GDM. The prevalence of GDM in India is $16.55 \%$. $^{3}$ Hence this study is undertaken for screening GDM in Indian Women, and effective treatment, which will minimize the pregnancy complications due to maternal hyperglycemia.

\section{METHODS}

This prospective cohort study was carried out in the department of obstetrics and Gynecology, Shri Sathya Sai Medical College \& Research Institute, Tamil Nadu, India from August 2015 to July 2016. 153 nondiabetic pregnant women of gestational age $24-28$ wks. presenting to the 
Antenatal outpatient department were selected during the study, excluding diabetes mellitus diagnosed prior to pregnancy. The antenatal women were screened for GDM by administering $50 \mathrm{~g}$ Glucose challenge test, irrespective of last meal taken. Patients with Screening test value of $>130 \mathrm{mg} / \mathrm{dl}$ at 1 hour were considered screening test positive and subjected to standard 2 hour, 75 grams oral Glucose Tolerance Test (OGTT). OGTT is done by administering $75 \mathrm{~g}$ oral glucose mixed in $200 \mathrm{ml}$ of plain water and three blood samples were drawn at 0,1 and 2 hrs and the results were tabulated. Among them GDM was diagnosed according to the American Diabetes Association Criteria. Antenatal women were screened for GDM after getting informed written consent. Women diagnosed as GDM were allotted appropriate treatment with diet alone or diet plus insulin therapy. All The screened women were followed up during the antenatal visits. Out of the 153 patients, 5 were lost to follow up. Hence the remaining 148 women were considered for the present study and the pregnancy outcome of this cohort Population was recorded.

The inclusion criteria are singleton pregnancies with gestational age 24 to 28 weeks, irrespective of age, parity or socioeconomic status.

The exclusion criteria are women who were known diabetic T1, T2, women suffering from very chronic illness, and multiple pregnancies.

\section{Statistical analysis}

The data collected were analyzed using SPSS software version 20. odds ratio, $\mathrm{P}$ value were estimated.

\section{RESULTS}

In this study 148 antenatal women were considered for screening GDM and the data were analyzed. 12 women were diagnosed as GDM by two step method. The prevalence of GDM in our study was $8.1 \%$.

Table 1: Prevalence of GDM.

\begin{tabular}{|lll|}
\hline No of cases & GDM & Prevalence \\
\hline 148 & 12 & $8.1 \%$ \\
\hline
\end{tabular}

The majority of the cases $41.66 \%$ were of age group 2630 yrs. The prevalence of GDM increases with age. Age $>25$ yrs was the most common indication for screening.

In the present study the prevalence of GDM was $41.6 \%$ in primigravida and the prevalence of GDM was $58.3 \%$ in multigravidae. Among the 148 cases, 12 cases were diagnosed as GDM by ADA criteria and the odds ratio = 22.2 and the $\mathrm{P}$ value $<0.001$ (significant).

All the women diagnosed as GDM were advised to follow diabetic diet. $41.66 \%$ cases required diet alone and $58.3 \%$ cases required diet along with insulin.
Table 2: Gravida and its correlation with GDM.

\begin{tabular}{|lll|}
\hline Gravida & No. of cases & Percentage \\
\hline Primi & 5 & $41.6 \%$ \\
\hline Multi & 7 & $58.3 \%$ \\
\hline Total & 12 & $100 \%$ \\
\hline
\end{tabular}

Table 3: Mode of treatment in GDM patients.

\begin{tabular}{|lll|}
\hline Treatment & No of cases & Percentage \\
\hline Diet alone & 5 & $41.66 \%$ \\
\hline Diet+ Insulin & 7 & $58.33 \%$ \\
\hline
\end{tabular}

In our study $33 \%$ cases had spontaneous Vaginal delivery. $8 \%$ cases had Emergency lower segment caesarean section and $58 \%$ cases had Elective LSCS. The most common indication for LSCS was previous caesarean section with GDM.

Table 4: Mode of delivery in GDM patients.

\begin{tabular}{|lll|}
\hline Mode & No of cases & Percentage \\
\hline Elective LSCS & 7 & $58.33 \%$ \\
\hline Emergency LSCS & 1 & $8.33 \%$ \\
\hline Vaginal Delivery & 4 & $33.33 \%$ \\
\hline
\end{tabular}

\section{DISCUSSION}

In the Indian context, screening for GDM is essential in all pregnant women, as Indian women have 11 fold increased risk of developing GDM compared with caucasian women.

In a study by Rajesh rajput et al, the prevalence of GDM was $7.1 \%$ and they concluded that prevalence of GDM increased with increasing age group. ${ }^{4}$

In a study by De Sisto CL et al, the prevalence of GDM was $9.2 \%$ and the GDM patients were of $>26$ years age group. 5

In our study the Glucose challenge Test is done by drinking of $50 \mathrm{~g}$ glucose solution and glucose levels are measured at 1 hour. The cut off point for screen positive is taken as $130 \mathrm{mg} / \mathrm{dl}$ instead of $140 \mathrm{mg} / \mathrm{dl}$, and a higher proportion of women are detected and the sensitivity is improved. If the level found are above these cut-off values, the Patient has to undergo an oral glucose tolerance test (OGTT) after an overnight fast before the diagnosis is made. This method was recommended by the fifth international workshop conference on GDM and by ADA. ${ }^{6}$

In a study by Van Leeuwen et al, 50g GCT had sensitivity of $74 \%$ and specificity of $77 \%$. In the present study, 50g GCT had sensitivity of $83.3 \%$ and specificity of $81.6 \%$ which is comparable to the study by Saleh et al, they had sensitivity of $88 \%$ and specificity of $84 \% .^{7,8}$ 
In a study by Qummry Ali Hindi et al, the GDM Women who underwent Elective LSCS was 55\% and Emergency LSCS $20 \%$ and $25 \%$ had Vaginal delivery. In the present study, $58.3 \%$ cases underwent Elective LSCS, $8.3 \%$ Emergency LSCS and $33.3 \%$ had Vaginal delivery. ${ }^{9}$

In a Cochrane review by Alwan et al, the authors compared women who were randomly assigned to receive oral medication versus Women randomly assigned to receive insulin, they found that women who received oral medication had a $54 \%$ decrease in the risk of LSCS, compared to women who received insulin. In a study by Katrien Benhalima et al, the modified 2 step screening strategy with GCT and clinical risk factors improved the diagnostic accuracy. ${ }^{10,11}$

As proved by earlier studies, in our study also the prevalence of GDM is $8.1 \%$ and majority of GDM patients were of age group 26-30 yrs. 58.3\% of GDM cases were multi gravidae. The increased risk factors for GDM were increased maternal age and previous history of GDM. In our study authors have employed universal screening strategy.

\section{CONCLUSION}

Diagnostic efficacy of two step method improved the detection of GDM, especially in the high prevalence group. More number of GDM is diagnosed with two step method. The fact that the first step of the two step approach can be done without the women being in a fasting state, can thus be an advantage. Hence we suggest that two step method can be used for universal screening in high prevalence group women. Further studies are needed to evaluate the efficacy of two step method for screening Gestational diabetes mellitus.

Funding: No funding sources

Conflict of interest: None declared

Ethical approval: The study was approved by the Institutional Ethics Committee

\section{REFERENCES}

1. Lee AJ, Hiscock RJ, Wein P, Walker SP, Permezel M. Gestational diabetes Mellitus: Clinical predictors and long term risk of developing type 2 diabetes: a retrospective Cohort study using survival analysis. Diabetes care. 2007;30(4):878-83.

2. Ferrara A, Increasing Prevalence of gestational diabetes Mellitus: a public health perspective. Diabetes care 2007 Jul;30(2):S141-S6.
3. Seshiah V, Balaji V, Balaji S, Sekar A, Sanjeevi, Green A. Gestational diabetes in India. J Assoc Physicians India. 2004;52:707-11.

4. Rajput R, Yadav Y, Nanda S, Rajput M. Prevalence of gestational diabetes Mellitus \& associated risk factors at a tertiary care hospital in Haryana. Indian $\mathrm{J}$ Med Res. April 2013;137(4):728-33.

5. De Sisto CL, Kim SY, Sharma AJ. Prevalence estimates of Gestational Diabetes in the united states, Pregnancy risk Assessment Monitoring system (PRAMS), 2007 - 2010. Prev Chronic Dis. 2014 Jun $19 ; 11$.

6. Metzger BE, Buchanan TA, Coustan DR, De Leiva A, Dunger DB, Hadden DR, et al . Summary and recommendations of the fifth international workshop-conference on gestational diabetes mellitus. Diabetes care. 2007 Jul 1;30(2):S251-60.

7. van Leeuwen M, Louwerse MD, Opmeer BC, Limpens J, Serlie MJ, Reitsma JB, et al. Glucose challenge test for detecting gestational diabetes mellitus: a systematic review. BJOG: An Intern J Obstetr Gynaecol. 2012 Mar;119(4):393-401.

8. Salleh MM, Ardawi, Path FRC, Hassan AN, Hassan SJ, Hussein M. et al, Screening for gestational diabetes Mellitus in Pregnant females. FRCOG. Saudi M J. 2000; 21(2):155-60.

9. Qummry AH, Zohair JG, Amira BK, Obeid D, Mian UF. Deliveries among diabetic females; a tertiary care experience Department of Gynaecology, Alnoor Specialist hospital, Makkah, Saudi Arabia - Al Ameen J Med Sc I. 2012;5(4):407-9.

10. Alwan N, Tuffnell DJ, West J. Treatments for gestational diabetes. Cochrane database of systematic reviews. 2009 July 8:(3).

11. Benhalima $\mathrm{K}$, Van Crombrugge $\mathrm{P}$, Moyson $\mathrm{C}$, Verhaeghe J, Vandeginste S, Verlaenen H, et al. A modified two-step screening strategy for gestational diabetes mellitus based on the 2013 WHO criteria by combining the glucose challenge test and clinical risk factors. J Clin Med. 2018 Oct;7(10):351.

Cite this article as: Srinivasan S, Palanisamy V. Screening for gestational diabetes mellitus by two step method and the pregnancy outcome: a study in Indian women. Int J Reprod Contracept Obstet Gynecol 2019;8:3502-4. 